\title{
Effects of Recombinant Human Brain Natriuretic Peptide in Patients with Acute Pulmonary Embolism Complicated with Right Ventricular Dysfunction Who Underwent Catheter-Directed Therapy
}

\author{
Xiangdong Meng, ${ }^{1} \mathrm{MD}$, Mingming $\mathrm{Fu},{ }^{2} \mathrm{MD}$, Jianjie Wang, ${ }^{3} \mathrm{MD}$ and Hui $\mathrm{Xu},{ }^{4} \mathrm{MD}$
}

\begin{abstract}
Summary
Acute pulmonary embolism (PE) remains a significant cause of cardiovascular morbidity and mortality worldwide. Brain natriuretic peptide (BNP) combined with catheter-directed therapy (CDT) may improve right ventricular (RV) dysfunction and stabilize hemodynamics in acute PE.

We retrospectively studied 159 patients with confirmed acute PE who were treated with CDT and admitted to the intensive care unit of our department between September 2016 and May 2020. The patients were divided into the control group and the rhBNP group based on whether to receive recombinant human BNP treatment (rhBNP) or not. The basic characteristics of the patients between the control group and the rhBNP group was systematically compared during admission and follow-up. Risk factors for all-cause mortality within 30 days were determined using multivariate logistic regression analysis.

Respiratory rate was found to be significantly lower in the rhBNP group than in the control group. Patients in the rhBNP group had significantly lower levels of white blood cell, C-reactive protein (CRP), D-dimers, troponin I, creatinine, and N-terminal (NT)-proBNP compared with those in the control group. Levels of tricuspid annular plane systolic excursion were significantly higher in the rhBNP group than in the control group. The percentage of patients with rehospitalization readmission due to PE differed significantly between the control group and the rhBNP group. On the basis of the multivariate regression analysis, CRP, creatinine, troponin I, and NT-proBNP were independent factors of all-cause mortality in 30 days.

rhBNP is effective in the treatment of patients with RV dysfunction caused by acute PE who underwent CDT, which may be an alternative treatment option for improving clinical prognosis.
\end{abstract}

(Int Heart J 2022; 63: 8-14)

Key words: Right heart failure, White blood cell, C-reactive protein, D-dimers, Troponin I, Inflammation

A cute pulmonary embolism $(\mathrm{PE})$ remains a significant cause of cardiovascular morbidity and mortality due to right ventricular (RV) dysfunction worldwide. ${ }^{1-4)}$ Patients with acute PE with compromised hemodynamics and right heart strain as diagnosed via pulmonary computed tomography angiography (CTA) may benefit from endovascular treatment. Specifically, patients with acute submassive PE as indicated by evidence of cardiac ischemia [elevated troponin and brain natriuretic peptide (BNP)] and right heart strain are candidates for endovascular treatment or systemic thrombolysis. ${ }^{5}$ Although systemic thrombolysis may reduce mortality, it is also associated with a higher risk of hemorrhagic complications including a $2 \%-5 \%$ risk of hemorrhagic stroke. ${ }^{6)}$ Catheter- directed therapy (CDT) that uses pharmacomechanical methods and low-dose thrombolytic infusion is an alternative to systemic thrombolysis. Recently, CDT has demonstrated efficacy in the treatment of RV dysfunction in trial populations, ${ }^{7)}$ with no increase in bleeding complications.

It is noteworthy that plasma concentrations of BNP are increased in cases of isolated acute or chronic RV dysfunction. ${ }^{8}$ Recombinant human BNP (rhBNP) is effective in the treatment of acute or chronic heart failure.") However, little is known about rhBNP treatment of acute RV dysfunction caused by severe PE. Inflammation is considered a risk factor for venous thromboembolism. The association between inflammatory markers and the severity of acute PE should be explored. ${ }^{10)}$

From the ${ }^{1}$ Department of Basic Medicine, Jiamusi University, Jiamusi, China, ${ }^{2}$ Department of Foreign Language, North Sichuan Medical College, Nanchong, China, ${ }^{3}$ Department of Immunology, Jiamusi University, Jiamusi, China and ${ }^{4}$ Department of Biochemistry and Molecular Biology, Jiamusi University, Jiamusi, China.

The present study was supported by basic scientific research projects of Heilongjiang Provincial Colleges and Universities (to X.M.) and Basic Research Fund on Universities of Heilongjiang Province [grant number 2019-KYYWF-1349 (to X.M.)]; the Heilongjiang Postdoctoral Science Foundation [grant number LBH-Z16252 (to M.F.)]; and Basic Research Fund on Universities of Heilongjiang Province [grant number 2018-KYYWF-0931 (to J.W.)].

Address for correspondence: Jianjie Wang, MD, Department of Immunology, Jiamusi University, Jiamusi 154007, Heilongjiang, China. E-mail: jmsdxjianjie @ 163.com or Hui Xu, MD, Department of Biochemistry and Molecular Biology, Jiamusi University, Jiamusi 154007, Heilongjiang, China. E-mail: 19782003 @ $163 . c 0 m$

Received for publication February 8, 2021. Revised and accepted July 5, 2021.

doi: 10.1536/ihj.21-086

All rights reserved by the International Heart Journal Association. 

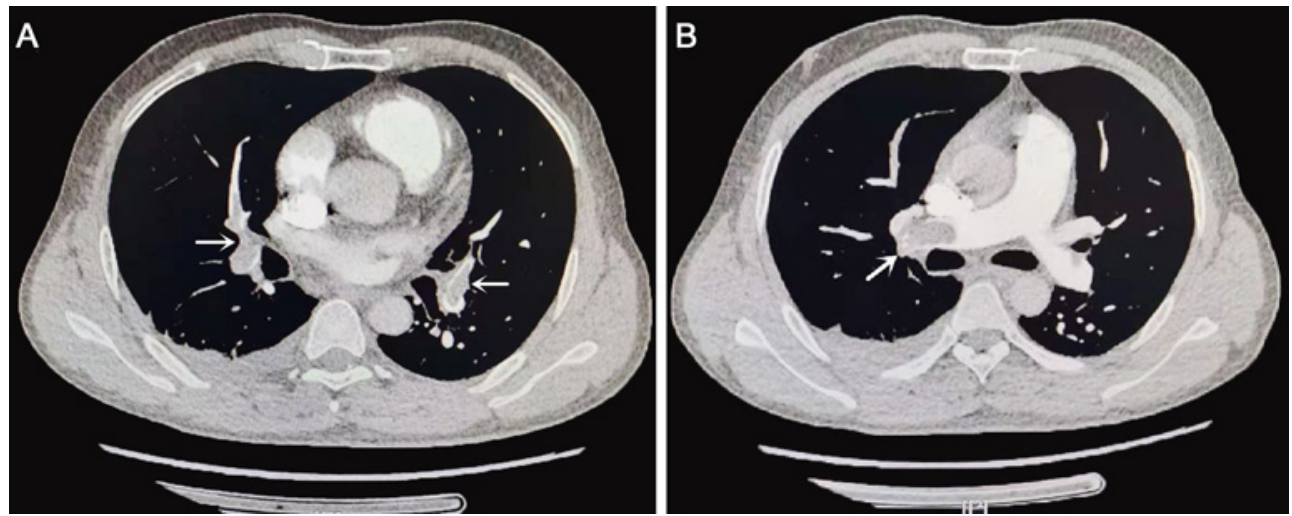

Figure 1. A 44-year-old male patient with high clinical suspicion of acute pulmonary embolism (PE). Axial images from a pulmonary arterial phase contrast-enhanced chest computer tomography. A: Bilateral subsegmental thrombus. B: Large, expansile, central thrombus in the right pulmonary artery.

Therefore, this study aims to determine how BNP levels are affected by acute PE with RV dysfunction and to test the clinical effect and prognostic implications of rhBNP in patients who underwent CDT by reducing the level of systemic inflammation.

\section{Methods}

Ethics statement: This study protocol conforms to the ethical guidelines of the 1975 Declaration of Helsinki as reflected in a priori approval by the institution's human research committee and the study was approved by the Research Ethics Committee of Jiamusi University, China. Informed consent was obtained from each patient.

Study population: We retrospectively studied 159 patients with confirmed acute PE who were treated with CDT and admitted to the intensive care unit of our department between September 2016 and May 2020. Patients were divided into the control group and the rhBNP group based on whether they received rhBNP or not. Patients were included if they had acute massive or submassive PE presenting within 14 days and pulmonary CTA evidence of proximal PE defined as a filling defect in at least one main or lobar pulmonary artery (Figure 1). Massive PE was defined as acute PE with sustained hypotension of systolic pressure $<90 \mathrm{mmHg}$ for at least 15 minutes or requiring inotropic support. Submassive PE was defined as acute PE causing RV dilatation and hypokinesis confirmed on echocardiography or pulmonary CTA, or both, without systemic hypotension. Patients were excluded if they were younger than 18 years, could not tolerate anticoagulant therapy, or had tumor thrombus in the pulmonary arteries.

Catheter-directed therapy: The decision about the ideal therapeutic strategy in an individual patient with acute PE should be made by a PE response team. ${ }^{11)}$ Massive PE was treated with immediate catheter-directed mechanical or pharmacomechanical thrombectomy using defined modern CDT techniques ${ }^{12)}$ including catheter-directed fragmentation of PE, intra-clot lytic injection, and aspiration (Figure 2). Submassive PE was treated with catheter-directed thrombolysis via low-dose hourly drug infusion into the clot with recombinant tissue plasminogen activator (0.5$1.0 \mathrm{mg} /$ hour) as a previously described protocol. ${ }^{13)}$ Thrombolytic drugs were delivered using standard infusion catheters such as the Unifuse (Angiodynamics, Queensbury, NY, USA) or multiside-hole pigtail catheter (Cook, Bloomington, IN, USA). Following completion of CDT, all patients received parenteral anticoagulation as a bridge to warfarin, an injectable anticoagulant as monotherapy, or rivaroxaban.

rhBNP treatment: After CDT, patients in the rhBNP group were given intravenous injection of a loading dose of rhBNP $1.5 \mu \mathrm{g} / \mathrm{kg}$ and they were given intravenous rhBNP $0.015 \mu \mathrm{g} / \mathrm{kg} /$ minute through an infusion pump for 72 hours, with a concentration of $10 \mu \mathrm{g} / \mathrm{mL}$.

Blood sampling: Blood samples were collected from cubital veins in all patients who were enrolled in the study. $\mathrm{N}$-terminal (NT)-proBNP and troponin I were measured on admission. The level of NT-proBNP in the plasma was measured using an Elecsys NT-proBNP analyzer, a commercially available electrochemiluminescent sandwich immunoassay (Roche Diagnostics GmbH, Mannheim, Germany). C-reactive protein (CRP) levels were measured using a commercially available immunonephelometric kinetic assay (BN ProSpec; Siemens, Tarrytown, NY, USA) using Cardiophase CRP reagents. Other biochemistry measurements were performed using the Jaffe kinetic method on a Hitachi 7600 Autoanalyzer (Hitachi, Ltd., Tokyo, Japan).

Statistical analysis: Quantitative variables were expressed as mean value \pm standard deviation, and qualitative variables were expressed as total number and percentage. The independent two-sample $t$-test or one-way analysis of variance with post hoc Student-Newman-Keuls test was used to assess the differences between multiple sets of data. Categorical variables were also compared using the chisquared or Fisher's exact test. Independent predictors of all-cause mortality within 30 days were identified using multivariate logistic regression analyses. Statistical significance was indicated when a two-sided $P$-value was < 0.05. All statistical analyses were performed using SPSS version 19.0 (SPSS Inc., Chicago, IL, USA). 

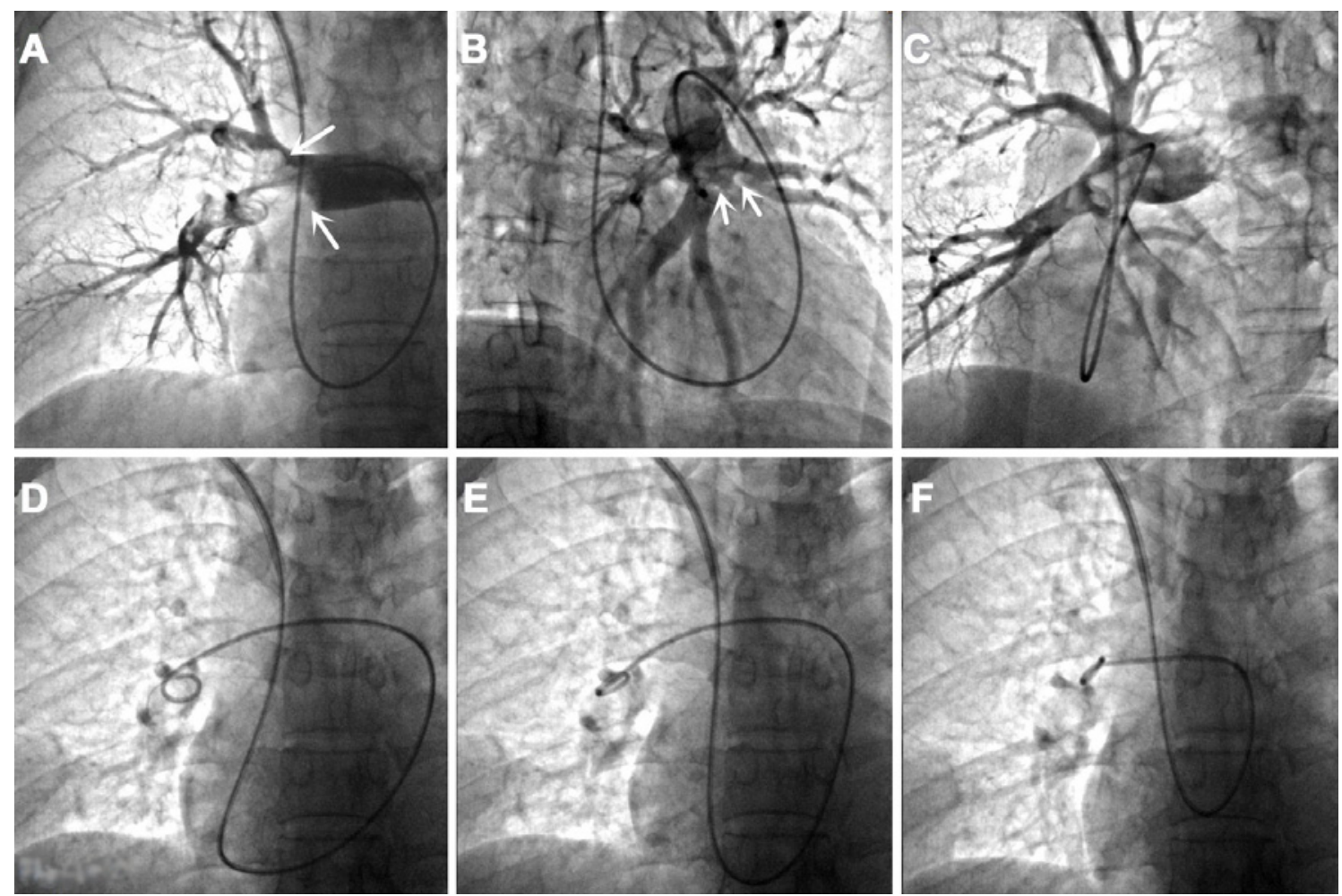

Figure 2. The process of catheter-directed therapy (CDT) in patients with acute pulmonary embolism (PE). A, B: Preliminary digital subtraction pulmonary angiography through a 9-Fr sheath positioned in the pulmonary artery via right internal jugular vein access demonstrates occlusive thrombus in the right pulmonary artery and scattered thrombi in the left pulmonary circulation, with no perfusion to the right lung. C: More lobar and segmental branch opacification and greater perfusion to the upper and lower lobes after CDT. D-F: Mechanical fragmentation is based on the insertion of a pigtail catheter into the pulmonary arteries via the femoral route.

\section{Results}

Baseline characteristics: One hundred and fifty-nine patients (71 men and 88 women) were enrolled. The baseline characteristics and invasive hemodynamic measurements of the control group and the rhBNP group are presented in Tables I, II. There were no significant differences in the baseline characteristics.

Comparison of patients' characteristics at discharge: Respiratory rate was found to be significantly lower in the rhBNP group than in the control group. Patients in the rhBNP group had significantly lower levels of white blood cell, CRP, D-dimers, troponin I, creatinine, and NTproBNP compared with those in the control group. Levels of tricuspid annular plane systolic excursion were significantly higher in the rhBNP group than in the control group (Table III).

Thirty days follow-up: During the follow-up period of 30 days, the incidence of readmission due to PE was significantly lower in the rhBNP group than in the control group (Table IV). Following multivariate logistic regression analysis, CRP [odds ratio $(\mathrm{OR})=1.256(95 \%$ confidence interval (CI) 1.104-1.430), $P=0.001)]$, creatinine $[\mathrm{OR}=1.033(95 \% \mathrm{CI} 1.002-1.065), P=0.039]$, NT-proBNP $[\mathrm{OR}=1.002(95 \%$ CI 1.001-1.004), $P=$ $0.005]$, and troponin I $[\mathrm{OR}=12.952(95 \%$ CI 1.529 109.684), $P=0.019$ ] were demonstrated to be independently associated with all-cause mortality within 30 days.
NT-proBNP $[\mathrm{OR}=1.004(95 \%$ CI 1.001-1.006), $P=$ 0.004] was demonstrated to be independently associated with death from PE within 30 days. (Table V).

\section{Discussion}

$\mathrm{PE}$ is the cause of $\leq 300,000$ deaths per year in the US, ranking high among the causes of cardiovascular mortality. ${ }^{14,15)}$ Of these patients, $34 \%$ died suddenly or within a few hours of the acute event, before therapy could be initiated or take effect. In recent years, increased use of more effective therapies and interventions, and possibly better adherence to guidelines, ${ }^{16,17)}$ has most likely exerted a significant positive effect on the prognosis of PE.

Acute RV dysfunction with resulting low systemic output is the leading cause of death in patients with highrisk PE. Experimental studies suggest that aggressive volume expansion is of no benefit and may even worsen RV function. ${ }^{18)}$ The principles of acute right heart failure management have been reviewed in a statement from the Heart Failure Association and the Working Group on Pulmonary Circulation and Right Ventricular Function of the European Society of Cardiology. ${ }^{19)}$ Systemic thrombolysis leads to faster improvements in pulmonary obstruction, pulmonary artery pressure (PAP), and pulmonary vascular resistance (PVR) in patients with PE, compared with unfractionated heparin alone; these improvements are accom- 
Table I. Baseline Characteristics of Patients with Acute Pulmonary Embolism and Right Ventricular Dysfunction

\begin{tabular}{lccc}
\hline & rhBNP group $(n=74)$ & Control group $(n=85)$ & $P$-value \\
\hline Age, years & $60.89 \pm 11.96$ & $61.88 \pm 10.40$ & 0.577 \\
Men, $n(\%)$ & $31(41.89)$ & $40(47.06)$ & 0.311 \\
Symptoms, $n(\%)$ & & & \\
Dyspnea & $53(71.62)$ & $56(65.88)$ & 0.273 \\
Chest pain & $17(22.97)$ & $24(28.24)$ & 0.283 \\
Syncope & $4(5.41)$ & $5(5.88)$ & 0.587 \\
Shock & $7(9.46)$ & $10(11.76)$ & 0.418 \\
Vital signs & & & \\
Systolic blood pressure, mmHg & $110.84 \pm 12.95$ & $108.45 \pm 11.98$ & 0.229 \\
Heart rate, beats/min & $91.43 \pm 7.37$ & $92.25 \pm 6.87$ & 0.472 \\
Respiratory rate, breaths/minute & $22.84 \pm 3.72$ & $22.92 \pm 4.69$ & 0.907 \\
Diagnosis, $n(\%)$ & & & \\
Pulmonary CTA & $54(72.97)$ & $64(75.29)$ & 0.439 \\
Echocardiography & $20(27.03)$ & $21(24.71)$ & 0.439 \\
Serum values & & & \\
WBC, $\times 10 \% / L$ & $13.36 \pm 4.76$ & $14.13 \pm 4.00$ & 0.267 \\
CRP, mg/L & $23.30 \pm 12.85$ & $26.12 \pm 10.80$ & 0.135 \\
D-dimers, $\mu \mathrm{g} / \mathrm{L}$ & $3416.30 \pm 2194.04$ & $3385.69 \pm 1955.79$ & 0.926 \\
Creatinine, $\mu$ mol/L & $121.19 \pm 43.52$ & $121.86 \pm 38.33$ & 0.918 \\
NT-proBNP, pg/mL & $3825.14 \pm 753.41$ & $3734.92 \pm 580.90$ & 0.396 \\
Troponin I, $\mu \mathrm{gg} / \mathrm{L}$ & $1.20 \pm 0.67$ & $1.22 \pm 0.74$ & 0.840 \\
Serum sodium, mmol/L & $142.18 \pm 1.54$ & $142.25 \pm 0.32$ & 0.687 \\
Echocardiographic parameters & & & \\
RV diameter, mm & $39.24 \pm 6.73$ & $39.58 \pm 7.71$ & 0.771 \\
RV hypokinesis, $n(\%)$ & $53(71.62)$ & $64(75.29)$ & 0.365 \\
RVSP, mmHg & $48.11 \pm 10.79$ & $44.66 \pm 11.48$ & 0.054 \\
TAPSE, cm & $2.05 \pm 0.85$ & $54(63.53)$ & 0.960 \\
Deep-vein thrombosis, $n(\%)$ & $45(60.81)$ & 0.385 \\
\hline
\end{tabular}

Mean values (standard deviation) and $\%(n)$ were reported for continuous and categorical variables, respectively. WBC indicates white blood cell; CRP, C-reactive protein; NT-proBNP, N-terminal pro-B type natriuretic peptide; CTA, computed tomography angiography; TAPSE, tricuspid annular plane systolic excursion; $\mathrm{RV}$, right ventricle; and RVSP, right ventricular systolic pressure.

Table II. Invasive Hemodynamic Measurements in Patients from the Catheter-directed Therapy

\begin{tabular}{|c|c|c|c|}
\hline & rhBNP group $(n=74)$ & Control group $(n=85)$ & $P$-value \\
\hline Pulmonary artery systolic pressure, $\mathrm{mmHg}$ & $42.95 \pm 15.26$ & $41.95 \pm 11.95$ & 0.647 \\
\hline Pulmonary artery diastolic pressure, $\mathrm{mmHg}$ & $17.57 \pm 8.60$ & $17.55 \pm 6.71$ & 0.990 \\
\hline Pulmonary artery mean pressure, $\mathrm{mmHg}$ & $25.27 \pm 7.79$ & $24.31 \pm 4.64$ & 0.338 \\
\hline Right atrial mean pressure, $\mathrm{mmHg}$ & $5.89 \pm 3.43$ & $6.16 \pm 2.65$ & 0.573 \\
\hline Right ventricular mean pressure, $\mathrm{mmHg}$ & $16.61 \pm 7.13$ & $17.69 \pm 5.28$ & 0.273 \\
\hline Pulmonary arterial oxygen saturation, $\%$ & $64.61 \pm 7.54$ & $64.34 \pm 4.18$ & 0.779 \\
\hline Cardiac output, L/minute & $5.13 \pm 1.31$ & $5.28 \pm 1.33$ & 0.498 \\
\hline Cardiac index, $\mathrm{L} / \mathrm{minute} / \mathrm{m}^{2}$ & $2.81 \pm 0.80$ & $2.78 \pm 0.97$ & 0.821 \\
\hline Pulmonary vascular resistance, wood & $3.00 \pm 2.07$ & $3.17 \pm 1.71$ & 0.549 \\
\hline
\end{tabular}

panied by a reduction in RV dilation on echocardiography. ${ }^{20,21)}$ However, systemic thrombolysis is accompanied by a significant risk of intracranial hemorrhage of $2 \%$ and general major hemorrhage of up to $20 \% .^{22,23)}$ CDT of acute $\mathrm{PE}$ is gaining increasing interest. ${ }^{724)}$ The rationale behind the removal of the thromboembolic burden in the pulmonary arterial circulation relates to improvement of RV impairment in the setting of elevated PVR and stabilization of the hemodynamics in the acute setting. ${ }^{25,26}$ Data from two prospective cohort studies, ${ }^{27,28)}$ and a registry, ${ }^{29)}$ with a total of 352 patients, support the improvement in RV function, lung perfusion, and PAP in patients with intermediate- or high-risk PE using systemic thrombolysis.
Conversely, medication is still the cornerstone of the treatment of RV dysfunction caused by acute PE. Studies have demonstrated that pathological BNP levels are frequently found in patients with RV dysfunction in acute PE. ${ }^{30)}$ BNP is secreted mainly from the cardiac ventricles in response to volume expansion and pressure overload. ${ }^{31)}$ Acute left ventricle (LV) dysfunction leads to a rapid increase in plasma BNP level. ${ }^{32}$ In published studies, plasma pro-BNP and BNP levels seem to be predictive of adverse clinical outcome in patients with acute $P E .^{33)}$ Therefore, rhBNP may be effective in the treatment of acute LV dysfunction through the mechanism of reducing pulmonary artery wedge pressure, which has been shown 
Table III. Comparison of Patients' Characteristics at Discharge

\begin{tabular}{lccc}
\hline & rhBNP group $(n=74)$ & Control group $(n=85)$ & $P$-value \\
\hline Duration of hospitalization, day & $5.3 \pm 0.5$ & $6.8 \pm 1.5$ & $<0.001$ \\
Vital signs & & & \\
Systolic blood pressure, $\mathrm{mmHg}$ & $109.27 \pm 12.46$ & $108.16 \pm 14.09$ & 0.603 \\
Heart rate, beats/minute & $85.54 \pm 3.79$ & $86.24 \pm 6.83$ & 0.439 \\
Respiratory rate, breaths/minute & $18.89 \pm 1.65$ & $20.69 \pm 4.84$ & 0.003 \\
Serum values & & & \\
WBC, $\times 10^{9} / \mathrm{L}$ & $8.24 \pm 2.12$ & $10.74 \pm 4.16$ & $<0.001$ \\
CRP, mg/L & $9.47 \pm 2.12$ & $16.23 \pm 7.97$ & $<0.001$ \\
D-dimers, $\mu \mathrm{g} / \mathrm{L}$ & $1359.77 \pm 735.23$ & $2364.95 \pm 1622.31$ & $<0.001$ \\
Creatinine, $\mu \mathrm{mol} / \mathrm{L}$ & $109.70 \pm 29.22$ & $134.07 \pm 59.75$ & 0.002 \\
NT-proBNP, pg/mL & $396.38 \pm 143.57$ & $1131.52 \pm 627.02$ & $<0.001$ \\
Troponin I, $\mu \mathrm{g} / \mathrm{L}$ & $0.200 \pm 0.154$ & $0.781 \pm 0.309$ & $<0.001$ \\
Serum sodium, mmol/L & $144.07 \pm 3.92$ & $144.52 \pm 3.81$ & 0.467 \\
Echocardiographic parameters & & & \\
RV diameter, mm & $36.58 \pm 6.26$ & $38.95 \pm 9.34$ & 0.067 \\
RV hypokinesis, $n(\%)$ & $57(77.03)$ & $74(87.06)$ & 0.143 \\
RVSP, mmHg & $40.85 \pm 7.61$ & $40.02 \pm 7.29$ & 0.485 \\
TAPSE, cm & $3.71 \pm 0.77$ & $3.30 \pm 0.78$ & 0.001 \\
\hline
\end{tabular}

Mean values (standard deviation) and \% $(n)$ were reported for continuous and categorical variables, respectively. WBC indicates white blood cell; CRP, C-reactive protein; NT-proBNP, N-terminal pro-B type natriuretic peptide; TAPSE, tricuspid annular plane systolic excursion; RV, right ventricle; and RVSP, right ventricular systolic pressure.

Table IV. In-Hospital and 30-Day Outcomes

\begin{tabular}{lccc}
\hline Feature, $n(\%)$ & rhBNP group $(n=74)$ & Control group $(n=85)$ & $P$-value \\
\hline In-hospital & & & \\
$\quad$ All-cause mortality & $6(8.11)$ & $13(15.29)$ & 0.221 \\
$\quad$ Death from PE & $4(5.41)$ & $8(9.41)$ & 0.384 \\
Within 30 days & & & \\
All-cause mortality & $8(9.41)$ & $15(17.65)$ & 0.263 \\
Death from PE & $6(8.11)$ & $10(11.76)$ & 0.599 \\
Rehospitalization associated with PE & $7(9.46)$ & $22(25.88)$ & 0.008 \\
New DVT within 30 days & $7(9.46)$ & $9(10.59)$ & 0.990 \\
\hline
\end{tabular}

Mean values (standard deviation) and \% (n) were reported for continuous and categorical variables, respectively. DVT indicates deep-vein thrombosis; and PE, pulmonary embolism.

Table V. Multivariate Logistic Regression Analysis for All-Cause Mortality and Death from PE Within 30 Days

\begin{tabular}{lcccc}
\hline & $\begin{array}{c}\text { All-cause mortality } \\
\text { Odds ratio }(95 \% \text { CI })\end{array}$ & $P$-value & $\begin{array}{c}\text { Death from PE } \\
\text { Odds ratio (95\% CI) }\end{array}$ & $P$-value \\
\hline WBC & $1.116(0.894-1.392)$ & 0.333 & $1.214(0.987-1.493)$ & 0.066 \\
CRP & $1.256(1.104-1.430)$ & 0.001 & $1.017(0.989-1.159)$ & 0.092 \\
D-dimers & $1.000(1.000-1.001)$ & 0.208 & $1.000(1.000-1.001)$ & 0.784 \\
Creatinine & $1.033(1.002-1.065)$ & 0.039 & $1.006(0.983-1.030)$ & 0.594 \\
NT-proBNP & $1.002(1.001-1.004)$ & 0.005 & $1.004(1.001-1.006)$ & 0.004 \\
Troponin I & $12.952(1.529-109.684)$ & 0.019 & $3.584(0.926-13.869)$ & 0.064 \\
Respiratory rate & $0.995(0.831-1.193)$ & 0.960 & $0.820(0.641-1.049)$ & 0.114 \\
TAPSE & $1.074(0.403-2.864)$ & 0.887 & $0.846(0.313-2.228)$ & 0.742 \\
Pulmonary artery mean pressure & $0.881(0.764-1.016)$ & 0.082 & $1.022(0.889-1.175)$ & 0.760 \\
\hline
\end{tabular}

PE indicates pulmonary embolism; CI, confidence interval; WBC, white blood cell; CRP, C-reactive protein; NT-proBNP, N-terminal pro-B type natriuretic peptide; and TAPSE, tricuspid annular plane systolic excursion.

in previous studies. ${ }^{34)}$

The therapeutic effect of rhBNP in the clinical management of cardiac diseases is well recognized, as well as its ability to exert anti-inflammatory activity in various organs. ${ }^{35)}$ rhBNP can increase the production of nitric oxide, which regulates the inflammatory factors, ${ }^{36)}$ as well as reduce acute lung injury in animal models. ${ }^{37)}$ In our study,
CRP levels in the rhBNP group were significantly reduced, and CRP was one of the independent causes of allcause mortality within 30 days. Several studies confirmed the inflammatory response in acute PE, suggesting the potential value of inflammatory markers in the diagnosis and prognosis in such cases. CRP was extensively studied in venous thromboembolism and had a sensitivity ranging 
$60 \%$ and $100 \%$ at the level between 5 and $10 \mathrm{mg} / \mathrm{L}$ and specificity varying between $52 \%$ and $78 \%{ }^{38)}$ Several studies reported that CRP had a sensitivity of $100 \%$ for excluding acute PE. ${ }^{39)}$ It was also found to have a prognostic value where it is associated with RV dysfunction, a predictor of worse outcomes in acute PE. ${ }^{40)}$ Inflammatory activation was directly related to the extent of both impairment of cardiac function and neurohormonal activation. CRP levels were inversely correlated with ejection fraction and directly correlated with BNP levels. ${ }^{41)}$ In a word, rhBNP showed anti-inflammatory and protective effects on RV dysfunction in acute PE.

Our results revealed that rhBNP significantly decreased the serum concentrations of TnI and NT-proBNP at discharge and incidence of readmission at follow-up. $\mathrm{BNP}$ is a natural factor against cardiac remodeling that regulates the levels of cytokines and inflammatory factors through regulating the expression of fibrosis genes. ${ }^{42}$ In addition, BNP selectively expands coronary and lung circulation, increases coronary blood flow, and reduces the consumption of myocardial oxygen. ${ }^{43)}$ Thus, we suspected that administration of rhBNP could further improve myocardial perfusion, limit myocardial impaired size, ameliorate cardiac dysfunction, and postpone ventricular remodeling in patients with PE undergoing CDT.

The present study has some limitations. First, the number of patients was relatively small. Second, there is a possibility of significant referral bias because of the retrospective and single-center design of the study. Third, data on long-term events and follow-up were relatively insufficient and are planned to be included in a future study.

In conclusion, rhBNP is effective in the treatment of patients with RV dysfunction caused by acute PE who underwent CDT, which may be an alternative treatment option for improving clinical prognosis.

\section{Disclosure}

Conflicts of interest: None.

\section{References}

1. Goldhaber SZ, Visani L, De Rosa M. Acute pulmonary embolism: clinical outcomes in the International Cooperative Pulmonary Embolism Registry (ICOPER). Lancet 1999; 353: 1386-9.

2. Ribeiro A, Lindmarker P, Juhlin-Dannfelt A, Johnsson H, Jorfeldt L. Echocardiography Doppler in pulmonary embolism: right ventricular dysfunction as a predictor of mortality rate. Am Heart J 1997; 134: 479-87.

3. Kasper W, Konstantinides S, Geibel A, Tiede N, Krause T, Just H. Prognostic significance of right ventricular afterload stress detected by echocardiography in patients with clinically suspected pulmonary embolism. Heart 1997; 77: 346-9.

4. Kasper W, Konstantinides S, Geibel A, et al. Management strategies and determinants of outcome in acute major pulmonary embolism: results of a multicenter registry. J Am Coll Cardiol 1997; 30: 1165-71.

5. Palm V, Rengier F, Rajiah P, Heussel CP, Partovi S. Acute pulmonary embolism: imaging techniques, findings, endovascular treatment and differential diagnoses. Rofo 2020; 192: 38-49.

6. Chatterjee S, Chakraborty A, Weinberg I, et al. Thrombolysis for pulmonary embolism and risk of all-cause mortality, major bleeding, and intracranial hemorrhage: a meta-analysis. JAMA 2014; 311: 2414-21.

7. Kucher N, Boekstegers P, Müller OJ, et al. Randomized, controlled trial of ultrasound assisted catheter-directed thrombolysis for acute intermediate-risk pulmonary embolism. Circulation 2014; 129: 479-86.

8. Nagaya N, Nishikimi T, Uematsu M, et al. Plasma brain natriuretic peptide as a prognostic indicator in patients with primary pulmonary hypertension. Circulation 2000; 102: 865-70.

9. Mills RM, LeJemtel TH, Horton DP, et al. Sustained hemodynamic effects of an infusion of nesiritide (human b-type natriuretic peptide) in heart failure: a randomized, double-blind, placebo-controlled clinical trial. Natrecor Study Group. J Am Coll Cardiol 1999; 34: 155-62.

10. Omar HR, Mirsaeidi M, Rashad R, et al. Association of serum albumin and severity of pulmonary embolism. Medicina (Kaunas) $2020 ; 56: 26$

11. Mahar JH, Haddadin I, Sadana D, et al. A pulmonary embolism response team (PERT) approach: initial experience from the Cleveland Clinic. J Thromb Thrombolysis 2018; 46: 186-92.

12. Kuo WT, Gould MK, Louie JD, Rosenberg JK, Sze DY, Hofmann LV. Catheter-directed therapy for the treatment of massive pulmonary embolism: systematic review and meta-analysis of modern techniques. J Vasc Interv Radiol 2009; 20: 1431-40.

13. Kuo WT. Endovascular therapy for acute pulmonary embolism. J Vasc Interv Radiol 2012; 23: 167-79.

14. Raskob GE, Angchaisuksiri P, Blanco AN, et al. Thrombosis: a major contributor to global disease burden. Arterioscler Thromb Vasc Biol 2014; 34: 2363-71.

15. Wendelboe AM, Raskob GE. Global burden of thrombosis: epidemiologic aspects. Circ Res 2016; 118: 1340-7.

16. Roy PM, Meyer G, Vielle B, et al. Appropriateness of diagnostic management and outcomes of suspected pulmonary embolism. Ann Intern Med 2006; 144: 157-64.

17. Jimenez D, Bikdeli B, Barrios D, et al. Management appropriateness and outcomes of patients with acute pulmonary embolism. Eur Respir J 2018; 51: 1800445.

18. Ghignone M, Girling L, Prewitt RM. Volume expansion versus norepinephrine in treatment of a low cardiac output complicating an acute increase in right ventricular afterload in dogs. Anesthesiology 1984; 60: 132-5.

19. Harjola VP, Mebazaa A, Celutkiene J, et al. Contemporary management of acute right ventricular failure: a statement from the Heart Failure Association and the Working Group on Pulmonary Circulation and Right Ventricular Function of the European Society of Cardiology. Eur J Heart Fail 2016; 18: 226-41.

20. Kline JA, Nordenholz KE, Courtney DM, et al. Treatment of submassive pulmonary embolism with tenecteplase or placebo: cardiopulmonary outcomes at 3 months: multicenter doubleblind, placebo-controlled randomized trial. J Thromb Haemost 2014; 12: 459-68.

21. Becattini C, Agnelli G, Salvi A, et al. Bolus tenecteplase for right ventricle dysfunction in hemodynamically stable patients with pulmonary embolism. Thromb Res 2010; 125: e82-6.

22. Kanter DS, Mikkola KM, Patel SR, Parker JA, Goldhaber SZ. Thrombolytic therapy for pulmonary embolism. Frequency of intracranial hemorrhage and associated risk factors. Chest 1997; 111: 1241-5.

23. Fiumara K, Kucher N, Fanikos J, Goldhaber SZ. Predictors of major hemorrhage following fibrinolysis for acute pulmonary embolism. Am J Cardiol 2006; 97: 127-9.

24. Konstantinides SV, Vicaut E, Danays T, et al. Impact of thrombolytic therapy on the long-term outcome of intermediate-risk pulmonary embolism. J Am Coll Cardiol 2017; 69: 1536-44.

25. Rali PM, Criner GJ. Submassive pulmonary embolism. Am J Respir Crit Care Med 2018; 198: 588-98.

26. Konstantinides SV, Meyer G, Becattini C, et al. 2019 ESC Guidelines for the diagnosis and management of acute pulmonary embolism developed in collaboration with the European Respiratory Society (ERS): the Task Force for the diagnosis and 
management of acute pulmonary embolism of the European Society of Cardiology (ESC). Eur Respir J 2019; 54: 1901647.

27. Piazza G, Hohlfelder B, Jaff MR, et al. A prospective, singlearm, multicenter trial of ultrasound-facilitated, catheter-directed, low-dose fibrinolysis for acute massive and submassive pulmonary embolism: the SEATTLE II study. JACC Cardiovasc Interv 2015; 8: 1382-92.

28. Tapson VF, Sterling K, Jones N, et al. A randomized trial of the optimum duration of acoustic pulse thrombolysis procedure in acute intermediate-risk pulmonary embolism: the OPTALYSE PE trial. JACC Cardiovasc Interv 2018; 11: 1401-10.

29. Kuo WT, Banerjee A, Kim PS, et al. Pulmonary embolism response to fragmentation, embolectomy, and catheter thrombolysis (PERFECT): initial results from a prospective multicenter Registry. Chest 2015; 148: 667-73.

30. Krüger S, Graf J, Merx MW, et al. Brain natriuretic peptide predicts right heart failure in patients with acute pulmonary embolism. Am Heart J 2004; 147: 60-5.

31. Maisel AS, Koon J, Krishnaswamy R, et al. Utility of Bnatriuretic peptide as a rapid, point-of-care test for screening patients undergoing echocardiography to determine left ventricular dysfunction. Am Heart J 2001; 141: 367-74.

32. Hama N, Itoh H, Shirakami G, et al. Rapid ventricular induction of brain natriuretic peptide gene expression in experimental acute myocardial infarction. Circulation 1995; 92: 1558-64.

33. Kucher N, Printzen G, Doernhoefer T, Windecker S, Meier B, Hess OM. Low pro-brain natriuretic peptide levels predict benign clinical outcome in acute pulmonary embolism. Circulation 2003; 107: 1576-8.

34. Michaels AD, Klein A, Madden JA, Chatterjee K. Effects of intravenous nesiritide on human coronary vasomotor regulation and myocardial oxygen uptake. Circulation 2003; 107: 2697701.
35. Li N, Jin H-X, Song Z, Bai C-Z, Gao Y. Protective effect of recombinant human brain natriuretic peptide on acute renal injury induced by endotoxin in canines. Cell Biochem Biophys 2014; 70: 1317-24.

36. Peng T, Shen E, Fan J, Zhang Y, Arnold JMO, Feng Q. Disruption of phospholipase Cgammal signalling attenuates cardiac tumor necrosis factor-alpha expression and improves myocardial function during endotoxemia. Cardiovasc Res 2008; 78: 90-7.

37. Yang H, Song Z, Jin H, Cui Y, Hou M, Gao Y. Protective effect of rhbnp on intestinal injury in the canine models of sepsis. Int Immunopharmacol 2014; 19: 262-6.

38. Maskell NA, Butland RJA. A normal serum CRP measurement does not exclude deep vein thrombosis. Thromb Haemost 2001; 86: $1582-3$.

39. Cooper TJ, Prothero DL, Gillett MG, et al. Laboratory investigation in the diagnosis of pulmonary thromboembolism. Q J Med 1992; 83: 369-79.

40. Abul Y, Karakurt S, Ozben B, Toprak A, Celikel T. C-reactive protein in acute pulmonary embolism. J Investig Med 2011; 59: $8-14$.

41. Morel O, Sauer F, Imperiale A, et al. Importance of inflammation and neurohumoral activation in Takotsubo cardiomyopathy. J Card Fail 2009; 15: 206-13.

42. Miao Z-L, Hou A-J, Zang H-Y, et al. Effects of recombinant human brain natriuretic peptide on the prognosis of patients with acute anterior myocardial infarction undergoing primary percutaneous coronary intervention: a prospective, multi-center, randomized clinical trial. J Thorac Dis 2017; 9: 54-63.

43. Chen HH, Martin FL, Gibbons RJ, et al. Low-dose nesiritide in human anterior myocardial infarction suppresses aldosterone and preserves ventricular function and structure: a proof of concept study. Heart 2009; 95: 1315-9. 